\title{
Importance of indoor dust biological ultrafine particles in the pathogenesis of chronic inflammatory lung diseases
}

\author{
Jinho Yang ${ }^{1}$, Yoon-Keun Kim ${ }^{1}$, Tae Soo Kang ${ }^{2}$, Young-Koo Jee ${ }^{2}$, You-Young Kim ${ }^{3}$ \\ ${ }^{1}$ Institute of MD Healthcare Inc., Seoul, Korea; ${ }^{2}$ Department of Internal Medicine, Dankook University College of Medicine, \\ Cheonan, Korea; ${ }^{3}$ Department of Allergy and Clinical Immunology, National Medical Center, Seoul, Korea
}

The role of infectious agents in the etiology of inflammatory diseases once believed to be non-infectious is increasingly being recognized. Many bacterial components in the indoor dust can evoke inflammatory lung diseases. Bacteria secrete nanometer-sized vesicles into the extracellular milieu, so-called extracellular vesicles (EV). which are pathophysiologically related to inflammatory diseases. Microbiota compositions in the indoor dust revealed the presence of both Gram-negative and Gram-positive bacteria. Escherichia coli is a model organism of Gram-negative Enterobacteriaceae. The repeated inhalation of $E$. coli-derived EVs caused neutrophilic inflammation and emphysema in a dose-dependent manner. The emphysema induced by $E$. coli-derived EVs was partially eliminated by the absence of Interferon-gamma or interleukin-17, suggesting that Thr and/or Thr cell responses are important in the emphysema development. Meanwhile, the repeated inhalation of Staphylococcus aureus-derived EVs did not induce emphysema, although they induced neutrophilic inflammation in the lung. In terms of microbial EV compositions in the indoor dust, genera Pseudomonas, Acinetobacter, Enterobacter, and Staphylococcus were dominant. As for the clinical significance of sensitization to EVs in the indoor dust, EV sensitization was closely associated with asthma, chronic obstructive pulmonary disorder (COPD), and lung cancer. These data indicate that biological ultrafine particles in the indoor dust, which are mainly composed of microbial EVs, are important in the pathogenesis of chronic lung diseases associated with neutrophilic inflammation. Taken together, microbial EVs in the indoor dust are an important diagnostic and therapeutic target for the control of chronic lung diseases, such as asthma, COPD, and lung cancer.

Keywords: Ultrafine particle, Particulate matter, Extracellular vesicle, Indoor dust, Inflammatory pulmonary disease

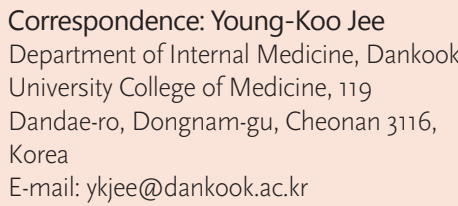

Correspondence: Young-Koo Jee Department of Internal Medicine, Dankook University College of Medicine, 119 Dandae-ro, Dongnam-gu, Cheonan 3116, Korea

E-mail:ykjee@dankook.ac.kr

Received: August 16, 2017 Accepted: November 16, 2017 Published: November 17, 2017

This article is available from: http://e-eht.org/

\section{INTRODUCTION}

Public concern for fine dust and ultrafine dust has recently been rising due to the influence of the yellow dust from China. Particulate matters are air pollutants that float in the air for a long period of time. Matter with a diameter of $10 \mu \mathrm{m}$ or less is considered suspended particulate matter, and matter with a di- ameter of $2.5 \mu \mathrm{m}$ or less is considered fine particulate matter. Ultrafine particles refer to particles of $100 \mathrm{~nm}$ or less; by their nature, these particles do not readily sediment or flocculate, and thus have a longer retention time in the atmosphere than fine particles allowing them to be carried long distances by the wind $[1,2]$. In terms of health hazards, fine particles are only absorbed by alveolar macrophages when they enter the lungs, whereas ul- 
trafine particles are also absorbed by airway epithelial cells, which can cause an inflammatory response in the airway. Moreover, unlike fine particles, ultrafine particles are absorbed into the body and increase the risk of cardiovascular and neuropsychiatric disease. On this basis, ultrafine particles are likely to be more harmful than fine particles [1-3]. The authors have recently confirmed that bacteria-derived extracellular vesicles (EV, nanovesicles), sized 50-100 $\mathrm{nm}$ are present in indoor environment. In the present commentary, we highlight evidence to confirm the indoor presence of bacteria-derived EVs corresponding to the ultrafine particles and aimed to explore the relationship between these bacteria-derived EVs and chronic inflammatory lung diseases.

\section{CHRONIC INFLAMMATORY LUNG DISEASES AND CAUSATIVE FACTORS}

Asthma is a global issue with a prevalence of $1-16 \%$ in children and adults worldwide. The prevalence of childhood asthma in Korea is 5-9\%, and is gradually increasing; the prevalence of asthma in adults has also been rising persistently, from $1.1 \%$ in 1998 to $3.1 \%$ in 2011 [4]. Since the prevalence of chronic obstructive pulmonary disorder (COPD) increases with age, the number of patients with COPD is expected to grow even further as we enter an increasingly aging society. COPD was the sixth leading cause of death worldwide in 1990, and is predicted to be the third leading cause by 2020 [5]. According to 2014 data from the Korean National Cancer Information Center, the incidence of lung cancer in Korea is the fourth highest among all cancers; it is the second highest for males and the fifth highest for females [6]. Lung cancer is associated with the highest mortality rate among all cancers for both males and females [6].

Smoking and air pollutants are known to be important in the development of chronic inflammatory lung diseases such as asthma, COPD, and lung cancer $[7,8]$. Three main causative factors have been suggested in a broad sense: chemical substances such as cigarette smoke and air pollution; protein antigens, including allergens secreted by house dust mites; and biological factors such as those derived from bacteria [9]. Exposure to chemical substances may cause chronic inflammatory diseases because of the inherent pharmacological or toxic effects of chemical substances. However, the etiology of a large proportion of chronic inflammatory diseases cannot be explained by these factors. Biological factors such as allergens, viruses, and bacterial factors can cause severe inflammatory reactions in some cases. A representative example of this is a hypersensitivity in the airway due to protein antigens, including allergens, virus- es, or bacterial factors. This is because our body is hypersensitive to protein antigens. Even if we are exposed to very small amounts, our body recognize the antigen as harmful and can cause an inflammatory reaction.

Clinical manifestations may vary depending on the type of inflammation that occurs in the airways. In the case of allergen, it is characterized by Th 2 cells-mediated inflammation and pathologically characterized by eosinophilic inflammation [10-12]. Eosinophilic inflammation usually responds well to anti-asthma medications such as inhaled corticosteroids. On the other hand, COPD is a disease characterized by neutrophilic inflammation and, immunologically, by Th17 cells-dependent inflammation. The most important causative factor of COPD is cigarette smoke, and no other causative factors have yet been identified. Notably, chronic inflammation is an important risk factor in the development of cancer. When we consider the fact that the incidence of lung cancer is increased in the presence of COPD regardless of smoking and the results of animal experiments, there is a limit to explain neutrophilic inflammation, which is a key pathology of severe asthma and COPD, only by cigarette smoke. Inflammation by Th17 cells is a typical defense mechanism against bacteria, and the possibility of exposure to biological causative factors derived from bacteria is attracting as a cause of Th17 hypersensitivity reaction and consequent neutrophilic inflammation.

\section{BACTERIA-DERIVED EXTRACELLULAR VESICLES, INDOOR BIOLOGICAL ULTRAFINE PARTICLE AS A NOVEL CAUSATIVE FACTOR}

Ultrafine particles exist both indoors and outdoors. There have been many reports on the aggravation and development of disease due to outdoor fine particulate matter. However, given that modern individuals spend $80-90 \%$ of the day indoors, the importance of indoor dust must not be overlooked. The ultrafine particles in the room are known to be caused by cooking, combustion, heating devices, tobacco smoke, biological sources, and human activity. Among them, biological fine particles contain microorganisms (bacteria, fungi, viruses) or organic compounds derived from microorganisms (endotoxin, serine proteases, etc.), and contribute to about $5-34 \%$ of indoor air pollution [13]. However, a scientific explanation of the mechanisms and the extent to which indoor contaminants including ultrafine particles causes diseases is still lacking. Microbes itself cannot be biological ultrafine particles because the size of bacteria is over a $\mu \mathrm{m}$, and its common health problem is causing an infection. Vi- 
ruses are only prevalent during epidemic seasons, and therefore this leaves the question of their pathogenic source as ultrafine particles, rather than as infectious agents, in the pathophysiology of lung diseases.

EVs secreted by bacteria are nanometer-sized $(50-100 \mathrm{~nm})$ substances for a purpose of communication between bacterial cells or eukaryotic cells, and are known to secrete a large amount during their proliferation or deaths $[14,15]$. The authors first reported the presence of a large amounts of bacteria-derived EVs in the indoor dust collected from bed in apartment [16-18]. These EVs were mainly derived from pathogenic bacteria. In terms of microbial EV compositions in the indoor dust, genera Pseudomonas, Acinetobacter, Enterobacter, and Staphylococcus were dominant. Bacteria-derived EVs in the indoor dust caused severe airway inflammation, which resulted not only in severe asthma but also in emphysema, which is a major cause of irreversible airway obstruction [16]. Repetitive inhalation of EVs derived from Staphylococcus aureus, which is a typical Grampositive bacterium, into the mice resulted in neutrophilic inflammation without emphysematous changes [17]. On the other hand, EVs derived from Gram-negative bacterium Escherichia coli caused neutrophilic inflammation accompanied by emphysema when repeatedly inhaled into the mice airway [18]. These results suggest that the pathogenicity of bacteria-derived EVs is dependent on the substances contained in the EVs.

In a clinical study evaluating the risk of indoor dust EVs isolated from apartment beds, only $5 \%$ of allergic rhinitis, atopic dermatitis and healthy children were sensitized to indoor dust EV. Whereas over $50 \%$ of childhood asthma were sensitized to indoor dust EV, suggesting that EVs present in the indoor dust could be an important causative factor in the pathogenesis of childhood asthma [16]. Moreover, anti-dust EV immunoglobulin $\mathrm{G}(\mathrm{IgG})$ antibody titers in serum were significantly higher in patients with non-eosinophilic asthma, COPD, and lung cancer than in healthy control subjects irrespective of age, sex or smoking history [19]. Adjusted multiple logistic regression revealed that sensitization to indoor dust EV (high serum anti-dust EV $\mathrm{IgG}$ ) was an independent risk factor for asthma (odds ratio [OR], 3.3), COPD (OR, 8.0), and lung cancer (OR, 38.7) [19]. These results signify that bacteria-derived EVs in the indoor dust are a major risk factor in the development of asthma, COPD, and lung cancer although further studies should be conducted.

\section{CONCLUSION}

In the aged society of the 21 st century where the life expectancy of human being is increased due to sanitization, antibiotics and vaccines, non-communicable chronic inflammatory diseases associated with immune dysfunction have become major diseases which determines the life span and quality of life [20]. Asthma, COPD, and lung cancer are very important chronic inflammatory lung diseases, and these diseases are increasingly prevalent along with aging population and changes in living environment. Increased exposure to causative factors from industrialization and urbanization is considered to be an important cause underlying the increase in the prevalence of these diseases. Notably, the indoor environment has changed dramatically as it has become increasingly shut off from the outside. Based on the knowledge that EVs derived from pathogenic bacteria are present in large volumes in the indoor environment, and given that these are important causative factors in asthma, COPD, and lung cancer, it is very important to avoid exposure to these bacteria and extracellular vesicles as a primary preventive measure against chronic inflammatory lung diseases. To this end, it is crucial to maintain an indoor environment that is not amenable to house dust mites, which are a major source of bacteria, as well as to make efforts to eliminate dust and provide sufficient ventilation.

\section{CONFLICT OF INTEREST}

The authors have no conflicts of interest associated with the material presented in this paper.

\section{ORCID}

\author{
Jinho Yang https://orcid.org/0000-0001-7207-6846 \\ Young-Koo Jee http://orcid.org/0000-0001-5800-8038
}

\section{REFERENCES}

1. Stone V, Miller MR, Clift MJ, Elder A, Mills NL, Møller P, et al. Nanomaterials versus ambient ultrafine particles: an opportunity to exchange toxicology knowledge. Environ Health Perspect 2017; 25(10):106002.

2. Karottki DG, Spilak M, Frederiksen M, Jovanovic Andersen Z, Madsen AM, Ketzel M, et al. Indoor and outdoor exposure to ultrafine, fine and microbiologically derived particulate matter related to cardiovascular and respiratory effects in a panel of elderly urban citizens. Int J Environ Res Public Health 2015;12(2):1667-1686.

3. Totlandsdal AI, Cassee FR, Schwarze P, Refsnes M, Låg M. Diesel exhaust particles induce CYP1A1 and pro-inflammatory responses via differential pathways in human bronchial epithelial cells. Part Fibre Toxicol 2010;7:41.

4. Korean Academy of Asthma, Allergy and Clinical Immunology. Korean guideline for asthma; 2015 [cited 2017 Nov 16]. Available from: http://www.allergy.or.kr/file/150527_01.pdf(Korean). 
5. Mathers CD, Loncar D. Projections of global mortality and burden of disease from 2002 to 2030. PLoS Med 2006;3(11):e442.

6. National Cancer Information Center. Cancer registry statistics 2014 [cited 2017 Nov 6]. Available from: http://www.cancer.go.kr/ $\mathrm{mbs} /$ cancer (Korean).

7. Jang AS. Particulate matter and bronchial asthma. Korean J Med 2015;88(2):150-155 (Korean).

8. Chiba H, Abe S. The environmental risk factors for COPD--tobacco smoke, air pollution, chemicals. Nihon Rinsho 2003;61(12): 2101-2106 (Japanese).

9. Brandt EB, Biagini Myers JM, Acciani TH, Ryan PH, Sivaprasad U, Ruff B, et al. Exposure to allergen and diesel exhaust particles potentiates secondary allergen-specific memory responses, promoting asthma susceptibility. J Allergy Clin Immunol 2015;136(2): 295-303.

10. Dowse GK, Turner KJ, Stewart GA, Alpers MP, Woolcock AJ. The association between Dermatophagoides mites and the increasing prevalence of asthma in village communities within the Papua New Guinea highlands. J Allergy Clin Immunol 1985;75(1 Pt 1):75-83.

11. Charpin D, Birnbaum J, Haddi E, Genard G, Lanteaume A, Toumi $\mathrm{M}$, et al. Altitude and allergy to house-dust mites. A paradigm of the influence of environmental exposure on allergic sensitization. Am Rev Respir Dis 1991;143(5 Pt 1):983-986.

12. Platts-Mills TA, Vervloet D, Thomas WR, Aalberse RC, Chapman $\mathrm{MD}$. Indoor allergens and asthma: report of the Third International Workshop. J Allergy Clin Immunol 1997;100(6 Pt 1):S2-S24.

13. Srikanth P, Sudharsanam S, Steinberg R. Bio-aerosols in indoor environment: composition, health effects and analysis. Indian J Med Microbiol 2008;26(4):302-312.
14. Lee EY, Bang JY, Park GW, Choi DS, Kang JS, Kim HJ, et al. Global proteomic profiling of native outer membrane vesicles derived from Escherichia coli. Proteomics 2007;7(17):3143-3153.

15. Lee EY, Choi DY, Kim DK, Kim JW, Park JO, Kim S, et al. Grampositive bacteria produce membrane vesicles: proteomics-based characterization of Staphylococcus aureus-derived membrane vesicles. Proteomics 2009;9(24):5425-5436.

16. Kim YS, Choi EJ, Lee WH, Choi SJ, Roh TY, Park J, et al. Extracellular vesicles, especially derived from Gram-negative bacteria, in indoor dust induce neutrophilic pulmonary inflammation associated with both Th1 and Th17 cell responses. Clin Exp Allergy 2013; 43(4):443-454.

17. Kim MR, Hong SW, Choi EB, Lee WH, Kim YS, Jeon SG, et al. Staphylococcus aureus-derived extracellular vesicles induce neutrophilic pulmonary inflammation via both Th1 and Th17 cell responses. Allergy 2012;67(10):1271-1281.

18. Kim YS, Lee WH, Choi EJ, Choi JP, Heo YJ, Gho YS, et al. Extracellular vesicles derived from Gram-negative bacteria, such as Escherichia coli, induce emphysema mainly via IL-17A-mediated neutrophilic inflammation. J Immunol 2015;194(7):3361-3368.

19. Kim YS, Choi JP, Kim MH, Park HK, Yang S, Kim YS, et al. IgG sensitization to extracellular vesicles in indoor dust is closely associated with the prevalence of non-eosinophilic asthma, COPD, and lung cancer. Allergy Asthma Immunol Res 2016;8(3):198-205.

20. World Health Organization. Noncommunicable diseases progress monitor 2017; 2017 [cited 2017 Nov 16]. Available from http:// www.who.int/nmh/publications/ncd-progress-monitor-2017/ en/. 